\title{
An Enhanced Packet Transmission Framework for Handling Real Time Burst Traffic in Wireless Sensor Networks
}

\author{
Sangeetha Ganesan ( $\square$ sangeetha.g@auist.net ) \\ Anna University Chennai https://orcid.org/0000-0002-7846-3529 \\ Vijayalakshmi Muthuswamy \\ Anna University Chennai
}

\section{Research Article}

Keywords: Congestion control, Torrent weight, Stochastic traffic generator, M-Pareto process, Heavy-tailed traffic

Posted Date: January 10th, 2022

DOl: https://doi.org/10.21203/rs.3.rs-1034809/v1

License: (c) (i) This work is licensed under a Creative Commons Attribution 4.0 International License.

Read Full License 


\title{
An Enhanced Packet Transmission Framework for handling Real Time Burst Traffic in Wireless Sensor Networks
}

\author{
G. Sangeetha \\ Dept. of Information Science \&Technology, Anna University, Chennai, INDIA \\ E-mail: sangeetha.g@auist.net \\ M. Vijayalakshmi \\ Dept. of Information Science \&Technology, Anna University, Chennai, INDIA \\ E-mail: vijim@auist.net
}

\begin{abstract}
Congestion control for real time traffic is an important network measure to be handled in case of repeated event triggers, continuous packet re-transmissions, node interference, node deaths and node failures in Wireless Sensor Networks (WSNs). Network modelling for transmission of packets from source node to sink using probabilistic M/Pareto and Poisson processes have been examined in the past. The existing methodologies are deficit in designing a queuing framework considering other network parameters such as energy consumption and delay for alleviating congestion and thereby efficiently routing packets to sink by reducing packet drops. To overcome this fall back, a Minimum Weight Estimation for Mitigating Congestion during Real Time Burst Traffic (MWCBT) framework is proposed. This gives a precautionary solution against heavy traffic occupancy among the interim and sink-neighbouring nodes in WSNs is proposed. Routing of packets using a congestion-free path is required to increase the node lifespan. An optimal M/Pareto stochastic traffic generator is used in combination with traffic factors such as energy and delay to predict amount of traffic across nodes. A simpler congestion prediction mechanism is performed to control the occurrence of heavy-tailed traffic distributions. A torrent weight value for incoming traffic is generated at each node periodically that directs routing of data packets to sink. The devised MWCBT framework supervises real-time traffic congestion and is found to be more optimal than the existing approaches for network traffic modelling. The proposed approach achieves greater packet delivery ratio and less node congestion compared to the existing network modelling techniques.
\end{abstract}

Keywords: Congestion control, Torrent weight, Stochastic traffic generator, M-Pareto process, Heavy-tailed traffic 


\section{INTRODUCTION}

Congestion control $[1,2]$ is a major factor to be considered in WSNs in case of repeated event triggering in an environmental area. When there is a disaster to occur such as landslide or tsunami, then the sensors are consecutively stimulated to receive and send response messages to the sink or Base Station (BS). There are a number of textual messages, captured multimedia images and video coverage sent to the sink simultaneously [31, 32]. The sensor nodes mostly resemble many to one node placement that further restricts the amount of response data sent to the sink. This leads to intense congestion on the intermediate sensor nodes and the nodes close to sink $[21,22]$. Under this situation, a number of sensitive data packets representing emergency occurrence are lost that may result in poor usage of sensor placement. So, there is a need to determine and restrict this congestion that occurs in the nodes causing packet drop, consecutive time delay and energy drain.

There are several approaches related to control congestion in WSNs for elastic and inelastic traffic types, event-to-sink and sink-to-event directional flow, source and network based, hopby-hop and multi-hop models. Most widely used methods include rate control and resource control operations. Fusion, PSFQ, ECODA, ESRT, TADR, STCP, TARA, LACAS are the standard congestion control algorithms [23,24] applicable for WSNs. Apart from these algorithms, there are certain approaches related to the stochastic process computation to alleviate congestion at nodes such as Markov Decision processes, Fluid models, Brownian Motion, Chi-Squared Distribution depending on discrete and continuous events.

In this paper, we consider the M/Pareto stochastic process for computing the expected number of data packets at each sensor node. The application of $\mathrm{M} /$ Pareto process as a model to handle broadband traffic is presented in [4]. The M/Pareto model is a family belonging to M/G/ queuing model. Long range dependence traffic [10] is handled using the Pareto process. In this model, queuing ability [25][29] depends on the level of aggregation within the burst process. In [5] Poisson Pareto Burst Process (PPBP) is discussed to be a simple and an accurate model for network traffic $[16,18]$. PPBP model is used to predict the queuing performance for multiplexing and link efficiency points. The author has demonstrated that the future optical Internet will be well-performing even if it is buffer less. PPBP is identified to use three measurable statistical parameters namely mean, variance and Hurst parameter. These parameters play an important role in teletraffic modelling [29,30]. 
The importance of the proposed work includes the practical application of M/Pareto process for queueing node congestion in WSNs. M/Pareto corresponds to a generalized M/G/ $\infty$ queue. Time delay is an important factor to be considered to communicate the packets from source node to sink. Another factor to be studied for efficient data coverage is the residual energy of sensors to remain alive. The two factors Time delay and Energy are integrated to compute the Torrent Weight during data congestion at each node [28]. The major objectives of MWCBT include:

- Usage of a stochastic process in the MWCBT framework for efficient queue management of incoming overflowed packets in WSN.

- Estimation of Torrent Weight for the incoming traffic flow using major traffic factors such as residual energy and delay probability.

- An optimal weighted node path to sink node is chosen based on the estimated minimal torrent weight.

- Prediction of a precise and congestion-independent path from source to sink nodes.

All the above-mentioned features lead to an increased performance for controlling congestion using an accurate path evaluation to reach sink node. The proposed MWCBT mechanism is a decentralized approach as packet transmission decisions are individually taken by network nodes. Depending on the computation of node weight, hop from one node to another occurs.

The remaining part of the paper is structured as follows: Section 2 presents the related works for shaping congestion control during transmission of real-time traffic from source to sink nodes. Section 3 illustrates the system design of MWCBT in detail. In Section 4, an elaborate discussion on the M/Pareto model is presented. The other traffic parameters related to $\mathrm{M} /$ Pareto process is shown in Section 5. Section 6 explains about the relationship between Energy Consumption and Time Delay. Torrent Weight (TW) is computed in Section 7. The performance analysis is represented in Section 8 where MWCBT is compared with Poisson traffic distribution and Pareto Traffic Modelling without Energy and Time Delay parameters. The proposed approach is concluded in Section 9.

\section{RELATED WORKS}

A realistic shaping of source burst traffic is done using the traffic generators Paretoon / Paretooff [6][11]. These generators produce burst length and division time. The traffic 
generators return the outcome of shape parameter $\alpha$. A mathematical model of burst traffic with their respective burst periods OFF and ON is computed. It is shown that a smooth continuous traffic exists for $\alpha$ between the ranges 1.5 to 2.0. The study of statistics of aggregation is focussed in [7]. The central limit theorem is shown to apply to traffic in networks and illustrates that as networks become larger, they carry traffic from free sources. M/Pareto model has sufficient parameters to substitute the basic Gaussian model. The effect of basic traffic characteristics such as peak, mean, burstiness and correlation in relation with overflow probability is considered. A mathematical illustration of how fractal traffic [7] is handled using M/Pareto and Gaussian models is delineated. Optical Burst Switching (OBS) networks also make use of blocking probability and mean delay with the burst arrival process considered to be Poisson [12] along with burst-length. The integral equations depicting the buffer motion are derived and solved for blocking probabilities and mean delays with burst size. A Level Crossing (LC) method [8] uses sample historical paths and enables studying the stochastic systems.

A generalization for data access patterns and long-term correlations related with clustering effects is given in [9]. Long-term correlations exhibit feasible estimates of traffic burst distribution taking into account the incident of node and link overloads [14][16]. This implies that neglecting long-term correlations lead to under estimation of empirical time under high node utilization. Three conditions must be satisfied to answer the extent till which long-term correlations can be taken: access patterns must be stationary, access rate allocation is close to Gaussian and system utilization is good below its greater throughput. The exponential shape parameter $q$, variation coefficient $\rho$ denoting inter-arrival with service times and Hurst exponent $\mathrm{H}$ denoting access rate change are essential for efficacy of the model. A pre-emptive multiple queue [20] using congestion removal mechanism for handling high traffic condition is proposed. This technique outperforms the traditional DropTail method in terms of throughput, packet drop and energy consumption. Varying thresholds for different drop probabilities were considered in this analytical model. However, the above technique has to be tested for increasing network size and queue length to expand efficiency.

Various ML techniques [35] can be utilized with WSNs that enables dynamically react to sudden changes in network. ML is a self-learning process from previous occurred experiences in network. Various ML-based MAC designs for WSN and data aggregation methods reliable on ML are presented. A statistical analysis of survey with network parameters is also presented. A congestion-aware data acquisition (CADA) mechanism [36] provides an alternative path to 
base station via Q-Learning for alleviating congestion. CADA initiates the identification of congested node where buffer occupancy ratio is higher. A resource control dependent CADA mechanism to mitigate congestion with parameters throughput, energy consumption, packet loss and latency are analyzed to predict congestion level in WSNs.

A multi-layer based MAC protocol named MLMAC-HEAP [37] is framed for harvesting solar energy considering throughput as the focused parameter. MLMAC-HEAP has a continuous supply of energy supplied from solar sources. The proposed model enhances performance by using fixed-capacity batteries. This model gives high throughput and outstands in comparison with other energy harvesting MAC protocols. Different queueing models needs to be explored for allocation in packet buffer with lossy channel utilization. A software-defined mobility management methodology [38] was analyzed solving CU planning and clustering problem. Least cost solutions for characterization of user mobility, signaling cost and system traffic have been addressed. The proposed solution functions better in contrast to LTE/NR networks. A backoff mechanism named TAT mechanism to support energy efficient communication between sensor nodes was devised on a heavily loaded network [39]. An analytical model was developed to influence their packet forwarding flow. There are four main techniques taken into consideration for performing high-speed packet I/O frameworks [40]. Memory pre-allocation, busy polling, zero copy and batch processing alternate costly operations in OS stack. An analytical model is designed for supporting packet forwarding flow. The statistical aspect of packet interarrival time has long-range dependency and multifractal statistical features. The distribution of incoming batch size follows Zipf's law.

The above existing mechanisms determined allowable number of packets that can be allocated into queue buffer but did not address other node parameters. Energy and delay are major parameters to be monitored at burst time to compute ideal node for next hop during packet transmission. A weighted value is assigned to each node to identify next hop node from current node data transmission. The computation of weighted value on a node includes permissible number of packets into buffer queue and individual node parameters.

Each model varies depending on the application and its utilization. Poisson distribution is wellsuited for applications consisting of light and separate traffic streams. Generalized Pareto distribution performs well for heavy traffic applications such as wireless multimedia sensor networks (WMSN). Weibull distribution is flexible distribution. Thus, Weibull distribution for designing over-voltage problems and across internet traffic. In this paper, we choose for 
Poisson Pareto burst process (PPBP) as it fulfils both long range dependence and selfsimilarity.

The scope of this paper is to design a M/Pareto process to compute the total number of allowed packets within buffer queue of a network node by examining energy and delay parameters. The length of buffer queue of each node is set up using starting point $\Phi p$ of pareto tail. Each node consists of pre-determined number of admissible packets occupied in buffer queue. When the node weight is computed at each node, the packets from source node transmit to minimum weighted node thereby reducing node congestion. Poisson or pareto distributions are already used as queueing models for data transmission to sink node. A combination of both distributions serves as a queueing model in MWCBT mechanism. The queueing model functions with network parameters such as energy and delay to fine tune queueing process. Thus, in traffic queueing model residual energy and delay are considered as major parameters in nodes to calculate probability value for torrent weight in nodes. Instead of calculating admissible number of packets into queue buffer, a node probability is computed apart from number of packets in buffer occupancy.

The novelty of the proposed MWCBT algorithm lies in finding a suitable node for next hop by using energy and delay variants to calculate torrent weight. The maximum number packets accommodated into node buffer is estimated using Poisson Pareto process thereby used in calculation of torrent weight. The node with minimum energy and delay values are chosen for next hop in network. The minimum weighted node is considered having less energy consumption and delay. In the proposed work, a less weighed node is considered to be efficient than a greater weighed node. A low weighed node denotes a congestion eliminated free-flow node for routing data packets. In traffic control models, the proposed MWCBT mechanism can be applied entirely taking into account node congestion to decide on next hop. On the other hand, in resource control algorithms an additional mechanism to lower or halt packet load supply from source sensor node has to be devised. So, there is an additional time complexity involved in resource control methods in combination with MWCBT mechanism.

\section{SYSTEM MODEL}

In the proposed MWCBT framework, we consider the M/Pareto stochastic process [18] as a queuing model to estimate the number of packets receiver a node can obtain at a time ' $t$ ' within a burst process from a source node [33][34]. Residual energy and delay probability of nodes 
are considered for estimating torrent weight for incoming traffic flows that decide the routing path for source packets to reach sink node. The queueing model uses an admissible number of packets that a node can receive with a calculated packet threshold. The proposed framework uses four parameters as basic inputs: $\lambda$ denotes the Poisson arrival rate, $r$ denotes the arrival rate of work to be done within an interval, $\Phi$ denotes the starting point of the Pareto tail and $\gamma$ denotes the rate of decay of Pareto tail. $\Phi$ is used to represent the minimum permissible burst length and is usually set to 1 representing all burst length duration lasting for one full timeperiod. The parameters $\Phi$ and $\gamma$ is set constant and the traffic arrival rate parameters $\lambda$ and $\mathrm{r}$ is varied within a time-slot.

The resultant number of packets in a node obtained using M/Pareto model is used to calculate the weight for congestion force at a particular node. There are two added factors namely, Energy $\left(E_{s}\right)$ and Time Delay $\left(D_{s}\right)$ that vary and affect the number of packets during transmission.

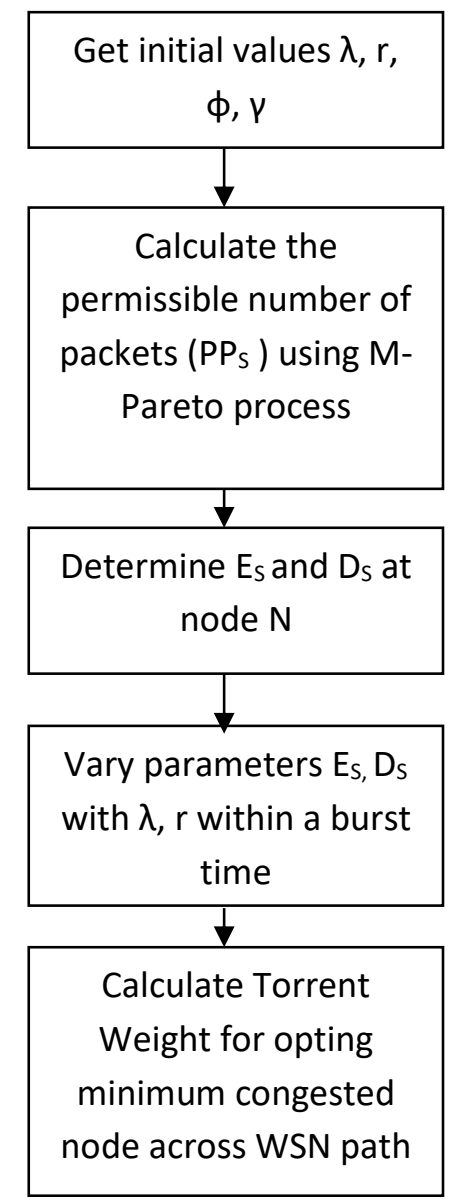

Fig 1. Steps for computing Torrent weight using MWCBT model

Fig. 1 narrates the organizational footsteps to select a less congested path across the network using the MWCBT model. 


\section{The M-Pareto Model}

To analyze the real-time traffic [17] in WSNs, M-Pareto model can be used as it can estimate probabilities and statistical analysis. M-Pareto is a Poisson process with overrunning bursts. Pareto distributions are used when there is an entire range from minimum to maximum values. In a normal Pareto model, there are three parameters considered namely the minimum value taken by random variable $\mathrm{x}_{\mathrm{min}}$, degree of density distribution $\alpha$ and number of packets involved in transmission $X$. The value limits include $\mathrm{X}_{\min }>0$ and $\alpha>0$. The Pareto curves are right skewed. If there exists a Pareto for random variable $\mathrm{X}$, then $\mathrm{X} \sim \operatorname{Pareto}\left(\mathrm{x}_{\min }, \alpha\right)$. The density function is given as,

$\mathrm{D}(\mathrm{x})=\left\{\begin{array}{c}\frac{\alpha x_{\min }^{\alpha}}{x^{\alpha+1}} \\ 0, \quad \text { otherwise }\end{array}, \mathrm{x}>x \min \right.$

The expected mean of the Pareto random variable is given as,

$\mathrm{E}(\mathrm{X})=\frac{\alpha X \min }{\alpha-1}$, where $\alpha>1$

The overall probability of Pareto distribution can be calculated using,

$\mathrm{P}(\mathrm{X}>\mathrm{x})=\left\{\begin{array}{c}1, x<x \min \\ \left(\frac{x \min }{x}\right)^{\propto}, x \geq x \min \end{array}\right.$

Assume a random variable $\mathrm{Y}$ to undergo a Pareto distribution with ( $\mathrm{Ym}, \alpha)$, where $\mathrm{Ym}$ is the minimum value taken by the random variable and $\alpha$ is the degree of packet dispatch. If Y Pareto(1500,2), on calculating expected packet overflow probability. As Y Y Ym,

$\left(\frac{Y m}{Y}\right)^{\propto}=\left(\frac{1500}{2000}\right)^{2}=0.75^{2}=0.56$

This is the expected probability at the sensor node that makes it reliable for packet transfer. Each node has a predicted probability that is updated for a particular time period of thirty seconds.

In the M-Pareto model, there are four parameters: $\lambda$ denotes the Poisson arrival rate, $r$ denotes arrival rate within an active burst, $\gamma$ denotes the drop of Pareto tail, $\varphi$ denotes starting point of Pareto tail and $\lambda$ manages the incoming bursts. The mean number of arrivals within one burst is $\frac{r \Phi \gamma}{\gamma-1}$. The variance of $\mathrm{M} /$ Pareto process is finite. The burst process follows a Poisson 
distribution with fixed rate burst intervals. From [6] for large t, the variance is given as $2 r^{2} \lambda *$ $\frac{(\Phi \gamma t 3-\gamma)}{(1-\gamma)(2-\gamma)(3-\gamma)}$. There is the use of Hurst parameter $\mathrm{H}$ for determining growth increase corresponding to $\mathrm{t}^{2 \mathrm{H}}$. $\mathrm{H}$ value is defined as $\frac{3-\gamma}{2}$, where $\mathrm{H}$ is asymptotically self similar with M/Pareto process. The parameters of M/Pareto process such as $\varphi$ and $\gamma$ are assumed to be fixed. $\mathrm{H}$ is unaltered when the values $\lambda$ and $\mathrm{r}$ are changed each time within a burst process.

\section{Traffic measures to M/Pareto Model}

The important characteristics of a WSN are packet losses, delay, jitter and residual energy due to heavy-tailed congestion [11][14]. A single server queue is considered for analyzing traffic queuing levels. The above-mentioned measures can be determined by probability statistical values with their respective expected mean and variance levels [19] as shown in Fig. 2. In this paper, we consider the packets arriving within a burst time period $\mathrm{B}_{\mathrm{t}}$. Packet loss is interpreted as the ratio of total number of packets in the transmission at an incoming burst time to actual packets successfully received. This packet loss ratio is an important parameter to be considered to analyze the network performance to identify packet drop rate. In general, packets arriving to the buffer when it is fully occupied are lost. The next required parameter is delay to study regarding the service time of each data packet sent from the source sensor node to sink. The devised framework involves queuing analysis with input service time. Time delay in our context is defined as the total round trip time a packet arrives from the source node till the packet being serviced to reach the sink. Here, we assume a parametric term called Torrent probability indicating the heavy packet flow condition from source to consecutive sensor nodes. This situation mainly occurs if there is an immediate event trigger and all the sensor nodes collect data information sending data packets to neighboring nodes simultaneously $[26,27]$.

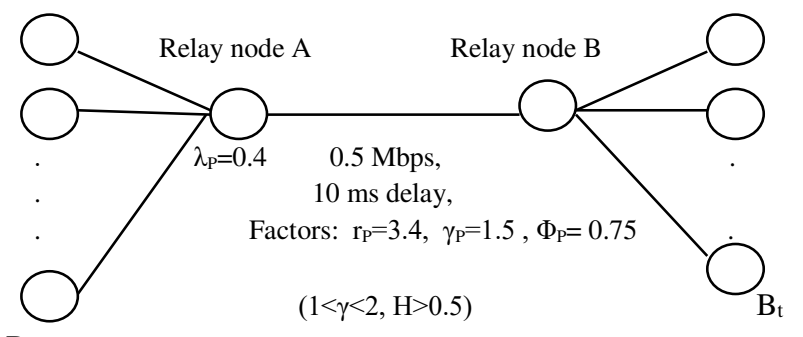

$\mathrm{B}_{\mathrm{t}}$

Fig.2. Network setup for traffic alleviation 
Probability of torrent weight is defined as the total packet buffer space to actual buffer occupied by packet flow. This probability is also called as the Overflow probability which is used to approximate packet loss ratio. Consider the incoming packet traffic to follow a large burst interval time. During this period, the torrent weight increases for the sensor nodes. Then the packet traffic has no bursts for a long-time which makes the probability small or null. If the incoming burst traffic increases, again the probability increases. The next traffic concerned parameter is the residual energy level of sensor nodes that is required to allow a successful packet transmission. Residual energy is defined as the ratio of sum between initial energy of all sensor nodes to total energy consumed by burst packet transmission. The Torrent probability is given in terms of M/Pareto stochastic process of heavy congestion prediction. From 2, the net amount of work arriving within ' $\mathrm{t}$ ' interval in the $\mathrm{M} /$ Pareto traffic model is given as,

$\mu=\mathrm{E}\left(\mathrm{A}_{\mathrm{n}}\right)=\frac{\lambda t r \Phi \gamma}{(\gamma-1)}$

The expected mean number of packets arriving at the sensor node $\mathrm{E}(\mathrm{P})$ is calculated. There are two network measure components added to our MWCBT framework namely Energy (E) and Time delay (TD). These variations are added without affecting the values of mean arrival rate of PBPP ( $\lambda$ ) and mean arrival rate of modeled stream (r). The mean number of packets allowed sensor node on receiving incoming traffic is given as,

$\mathrm{E}(\mathrm{P})=\frac{\lambda r \Phi \gamma}{(\gamma-1)}$

\section{Lemma 1: Let $\mathrm{E}(\mathrm{An})$ be the net amount of work in a M/Pareto process with the burst duration parameters $\gamma, \Phi$, then this process is applicable to MWCBT only if $\mathrm{E}(\mathrm{An})=$ $\mathbf{E}(\mathbf{P s})$.}

Proof. Consider the M/Pareto process of MWCBT with parameters $\lambda_{p}, r_{p}, \gamma_{p}, \Phi_{p}$ denoting the poisson burst arrival rate, active burst arrival rate, rate of decrease of pareto tail and starting point of pareto tail.

Assume MWCBT uses a Single Server Queue (SSQ) and follows a M/G/œ queuing model. Let the arrival times be denoted as $\left\{\alpha_{\mathrm{p}}: \alpha_{\mathrm{p}} \varepsilon \mathrm{R}\right.$, where $\left.\mathrm{p}=0,1,2, \ldots\right\}$ and the departure times be denoted as $\left\{\eta_{p}: \eta_{p} \varepsilon R\right.$, where $\left.p=0,1,2, \ldots\right\}$; R denotes the set of real numbers and $R^{+}$denotes the set of non-negative real numbers. $\mathrm{B}_{\mathrm{T}}$ denotes the burst time increasing or decreasing by one at a time $\mathrm{t}=\mathrm{y}_{\mathrm{p}}$.

Here, $d_{p}$ is the duration of the $p^{\text {th }}$ burst. For all $d_{p} \varepsilon R^{+}, y_{p}=\alpha_{p}+d_{p}$. The burst time $B_{T}$ is defined as,

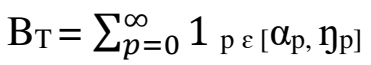


where, $1_{\mathrm{p}}=\left\{\begin{array}{l}1, \text { if } p \text { is true } \\ 0, \quad \text { otherwise }\end{array}\right.$

From (5), $\Phi>0$, for $1<\gamma<2, \mathrm{E}\left(\mathrm{d}_{\mathrm{p}}\right)=\frac{\Phi \gamma}{(\gamma-1)}$ and $\operatorname{Var}\left(\mathrm{d}_{\mathrm{p}}\right)=\infty$.

Consider a continuous time process ' $\mathrm{C}_{\mathrm{t}}$ ' representing the total amount of work done by all sensor nodes within a period $[0, t]$ at a constant rate ' $r$ '.

$\mathrm{Ct}=\mathrm{r} \int_{0}^{t} B T d t$

Therefore, mean is given by $\mathrm{E}\left(\mathrm{C}_{\mathrm{t}}\right)=\frac{\lambda \operatorname{tr} \Phi \gamma}{(\gamma-1)}$

Choose an arbitrary value $\theta$ in fixed time slots. Taking a positive value as $\theta=1$. Define the discrete time process as $\mu=\mathrm{E}\left(\mathrm{P}_{\mathrm{s}}\right)$ and $\sigma^{2}=\operatorname{Var}\left[\mathrm{P}_{\mathrm{s}}\right]$ representing the mean and variance of the number of packets received at the sensor node. The overall expected mean for the incoming packets at the sensor node is given as $\mu=\mathrm{E}\left(\mathrm{P}_{\mathrm{s}}\right)=\frac{\lambda r \Phi \gamma}{(\gamma-1)}$ at time slot $\theta$. This resembles ( $)$. Thus, the expected mean for MWCBT is the same as the expected mean for M/Pareto process such that $\mathrm{E}(\mathrm{An})=\mathrm{E}\left(\mathrm{P}_{\mathrm{S}}\right)$. The variance term and the Hurst condition $\mathrm{H}=\frac{(3-\gamma)}{2}$ for self-similarity is negligible and not opted.

\section{Relation between Energy and Delay parameters}

Residual energy in sensor nodes is a principal factor that has to be valued for computing torrent weight. Energy holes may occur in WSN when nodes drain their residual energy and become dead during packet transfer. If the next hop from source node is falling on the energy hole where there are dead nodes, then this decreases data transmission thereby multiplying packet drops and residual energy of the network. This serves the reason behind taking residual energy parameter into concern. Residual energy in the sensor nodes increases when the time delay component is less or null [3]. Energy decreases when the time delay component increases. For example, when the initial energy is $2 \mathrm{~J} / \mathrm{s}$ and there occurs a time delay of $3 \mathrm{~s}$ on a normal time interval of $3 \mathrm{~s}$, then the residual energy $\mathrm{E}_{\mathrm{s}}$ in the present sensor node is $0.21 \mathrm{~J} / \mathrm{s}$. Hence, residual energy is inversely proportional to delay in packet transmission [3].

$$
\begin{aligned}
& E_{S} \propto \frac{1}{D_{S}} \\
& \mathrm{D}_{\mathrm{s}}=1-\left(\frac{T i}{T i+T d}\right)
\end{aligned}
$$

$\mathrm{T}_{\mathrm{i}}$ denotes the initial Round Trip Time (RTT) for servicing a single packet within a burst in a sensor node. Td denotes the time delay [17] in servicing data packets from source to current sensor node. Let $\mathrm{D}_{\mathrm{s}}$ denote the total time delay that has occurred in the sensor node. Assume, $\mathrm{T}_{\mathrm{i}}=4 \mathrm{~s}$ and $\mathrm{T}_{\mathrm{d}}=2 \mathrm{~s}$ then time delay is given as, 
$\mathrm{D}_{\mathrm{s}}=1-\left(\frac{4}{4+2}\right)=0.3 \mathrm{~s}$

$\mathrm{E}$ is calculated in terms of Joule/second. $\mathrm{E}$ is defined as the difference between the initial amount of energy in the sensor node and total energy utilized for data packet transmission. The Torrent weight $\left(\mathrm{TW}_{\mathrm{S}}\right)$ is defined as the net congestion value at the particular sensor node. The lesser TW value node marks the more congestion-free and reliable path that is chosen. So, the minimal weighted sensor node is to be selected to achieve more efficient path with minimal delay and less energy consumption.

Node delay is the result of interference of various incoming packets in a node exceeding packet service ratio. When incoming packets reach the maximum threshold of a node buffer, congestion state occurs. This inference results in node congestion over a period of time. For free-flow of packets in a node, various mechanisms such as source node control, resource control and traffic diversion are utilized. Delay in nodes causes gradual energy drain leading to node deaths in network. The term traffic is related to node congestion caused due to excess delay in buffer service, energy holes in network due to more node failures. Hence, residual energy in nodes is inversely proportional to delay during packet transmission. Both energy and delay in nodes are related to packet traffic in this relation.

\section{Proposed MWCBT Framework}

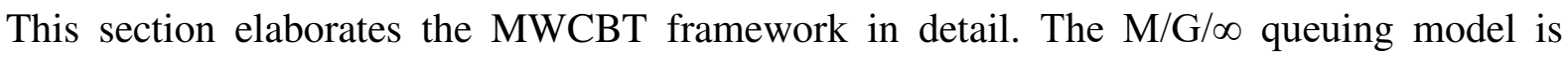
adopted for proposed technique to monitor network traffic. Residual energy and delay are the two parameters considered for each node within a possible packet arrival rate at any burst time interval $\mathrm{B}_{\mathrm{t}}$.

\subsection{Design of MWCBT framework}

\section{Algorithm for MWCBT Routing}

$\mathrm{P}_{\mathrm{s}}$ denotes M/Pareto process of MWCBT

$\lambda_{p}, r_{p}, \gamma_{p}, \Phi_{p}$ : parameters of poisson burst arrival rate, active burst arrival rate, rate of decrease of pareto tail and starting point of pareto tail.

$\mathrm{M} / \mathrm{G} / \infty$ is the queueing model assumed. The number of sensor nodes is denoted as $\mathrm{N} \varepsilon\left\{\mathrm{N}_{1}, \mathrm{~N}_{2}\right.$, $\left.\mathrm{N}_{3}, \ldots, \mathrm{N}_{\mathrm{n}}\right\}$

Es, Eres: Actual energy required by MWCBT and residual energy at node N

Ds, Dtot: Actual time delay due to MWCBT and total time delay at node N

Step 1: Obtain initial values for the parameters $\lambda_{\mathrm{p}}, \mathrm{r}_{\mathrm{p}}, \gamma_{\mathrm{p}}, \Phi_{\mathrm{p}}$

Step 2: Calculate the expected number of packets $\left(P_{s}>0\right)$ using the M/Pareto process. 
Step 3: Determine the residual energy level $E_{\mathrm{s}}$ at each node as the ratio of energy required for the incoming packets to the total energy of the current node $N$. Determine the time delay $D_{s}$ at node N as Round Trip Time (RTT) of incoming source packet.

Step 4: Interchange parameters Es, Ds with $\lambda$ and $r$ within a burst time ' $\mathrm{B}_{\mathrm{t}}$ '.

Step 5: Compute the Torrent Weight $\left(\mathrm{TW}_{\mathrm{s}}\right)$ and select minimum weighted path from intermediate source nodes to reach sink such that TWs $=E_{s}\left(P_{s}\right) *\left(\frac{D s}{\text { Eres }}\right)$

Step 6: If Es $>$ Eres choose the next adjacent path where Es $<=$ Eres. If Ds $>$ Dtot will result in choosing the next adjacent path $\mathrm{Ds}<=\mathrm{D}_{\text {tot }}$. This implies that if Es $>$ Eres or Ds $>$ Dtot then the current node is deleted from the congestion-free path to sink.

Step 7: Repeat steps 5 and 6 for each $\lambda$ and $r$ within every $B_{t}$.

Step 8: When Ws=0, then sink reached else follow step 7 till sink is reached.

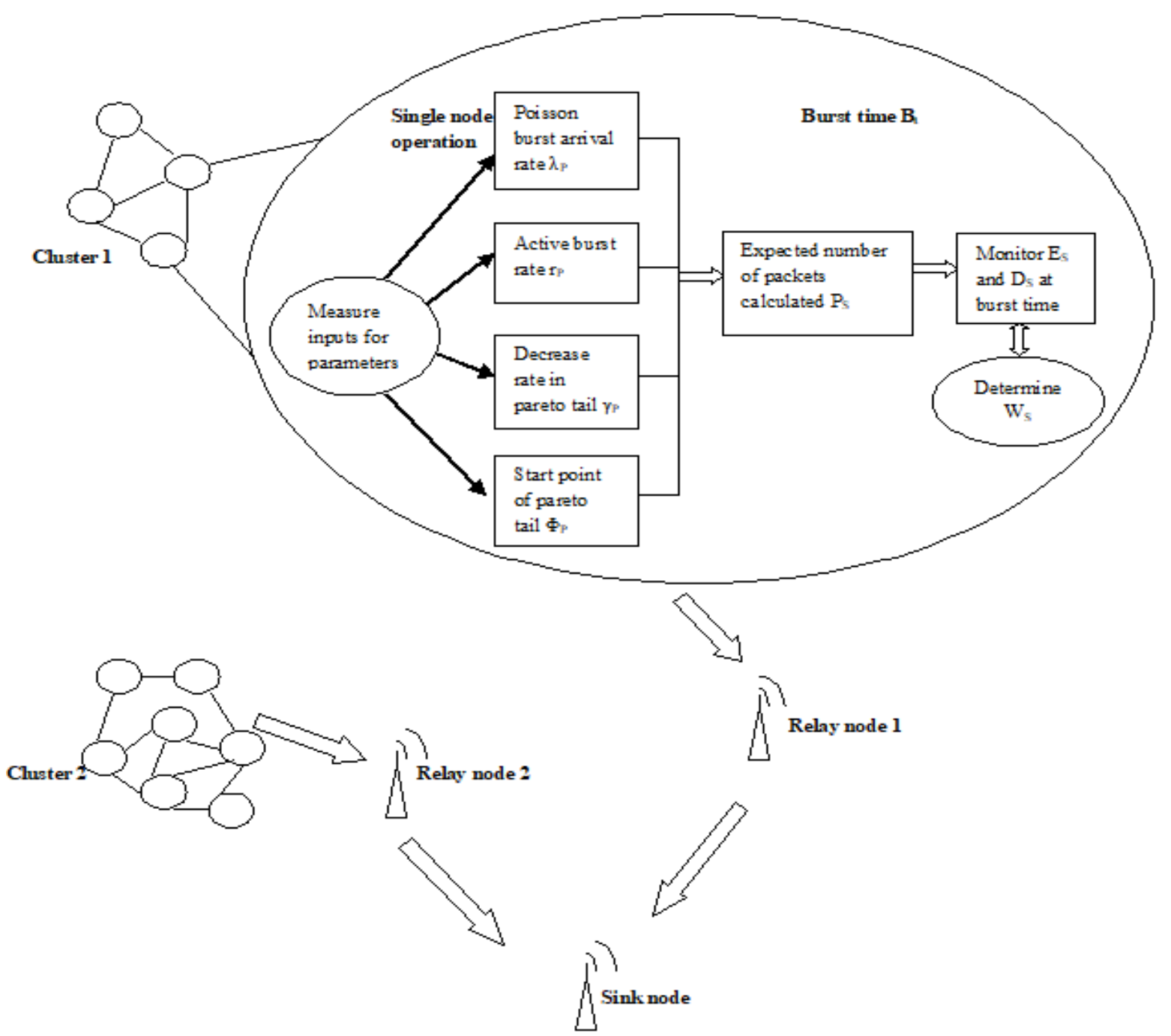


Fig.3. Operation of MWCBT framework

The comprehensive functioning of MWCBT prototype is projected in Fig. 3 and its gradual working is given in section 6.1.

\subsection{Prediction of Torrent Weight $\left(\mathrm{TW}_{\mathrm{s}}\right)$}

The below context describes an example of computing $\mathrm{TW}_{\mathrm{s}}$ across a network path. Assume the initial values for $E=1000 \mathrm{~J} / \mathrm{ms}$ at first node. Let the incoming packet arrival rate of PPBP is $\lambda=2$, mean arrival rate of incoming rate $r=3$, the number of packets within a single burst is $\varphi=1000$ packets/ms. Let the density of packet distribution $\gamma=2$. The mean number of packets allowed in sensor node is given as,

$\mathrm{PP}_{\mathrm{S}}=\mathrm{E}(\mathrm{P})=(2 * 3 * 1000 * 2) / 1$

$\mathrm{PP}_{\mathrm{S}}=12000$ packets/s

Let $\mathrm{PP}_{\mathrm{S}}$ denote the permissible number of packets in a node. Adding residual energy $\mathrm{E}_{\mathrm{res}}$ and delay $\mathrm{D}_{\mathrm{s}}$ components to calculate the Torrent Weight $\left(\mathrm{TW}_{\mathrm{s}}\right)$. $\mathrm{D}_{\mathrm{s}}$ denotes the calculated delay probability between time taken for source node to receive incoming packets and transmit it to next hop receiving node.

$$
\begin{aligned}
& \mathrm{TW}_{\mathrm{s}}=\mathrm{E}(\mathrm{P}) *\left(\frac{\mathrm{Ds}}{\text { Eres }}\right) \\
& \mathrm{E}_{\text {res }}=\mathrm{E}_{\text {init }}-\mathrm{E}_{\text {used }}
\end{aligned}
$$

If there is a delay of $T_{d}=2 s$, the delay probability at sensor node is given as

$\mathrm{D}_{\mathrm{s}}=1-\left(\frac{3}{3+2}\right)=0.4$

The residual energy $\mathrm{E}_{\text {res }}$ is given as the difference between initial energy and the utilized energy in the sensor node.

$\mathrm{TW}_{\mathrm{s}}=12000 *\left(\frac{0.4}{1000}\right)$

Therefore, $\mathrm{TW}_{\mathrm{s}}=4.8$ 
This is the Torrent Weight at current positioned sensor node. The minimum weighted node is to taken for more accuracy. The largest weighed node is the least accurate node that can be taken across sensor node path. This path will be definitely prone to maximum congestion with poor network measures.

\section{Lemma 2:}

\section{Determining Torrent Weight value approach is a Greedy-search algorithm.}

Let $\mathrm{A}_{\mathrm{T}}$ and $\mathrm{A}_{\mathrm{G}}$ denote the Torrent Weight approach and Greedy-search approach respectively. The number of vertices is $\mathrm{v}=1,2, \ldots \mathrm{N}$ and the number of edges is $\mathrm{e}=1,2, \ldots, \mathrm{M}$. Let Gr-(e,v) denote the edge and Gr-w(e,v) denote the weight in the computation of Greedy algorithm, distG denotes the array of distance from source node 's' to each node in graph ' $G$ '.

In $\mathrm{AG}, \operatorname{dist}_{\mathrm{G}}(\mathrm{s})=0, \operatorname{dist}_{\mathrm{G}}(\mathrm{v})=\infty, \mathrm{Q}$ represents queue data structure and $\mathrm{S}$ is an empty set.

In $\mathrm{A}_{\mathrm{T}}$ approach, when $\mathrm{Q} \neq$ empty, pop node $\mathrm{v} \neq \mathrm{s}$ from $\mathrm{Q}$ with smallest $\operatorname{dist}(\mathrm{v})$; add $\mathrm{v}$ to $\mathrm{s}$, indicating $\mathrm{v}$ as visited and update dist values. If for any adjacent node 'e', $\sum_{\substack{N-1, v=1 \\ N-1}} \operatorname{distG}(\mathrm{v})+$ Weight $(\mathrm{e}, \mathrm{v})<\operatorname{distG}(\mathrm{e})$ For all $\sum_{1}^{N-1} e$ update $\operatorname{distG}(\mathrm{e})$ or no updates done in $\operatorname{distG}(\mathrm{e})$. The dist $_{\mathrm{G}}$ is the shortest path tree from ' $\mathrm{s}$ '.

Assume, T-(e,v) denoting the edge and T-w(e,v) denoting the weight in the Torrent weight approach.

$\operatorname{dist}_{\mathrm{T}}(\mathrm{s})=\operatorname{dist}_{\mathrm{G}}(\mathrm{s})=0 ; \operatorname{dist}_{\mathrm{T}}(\mathrm{v})=\operatorname{dist}_{\mathrm{G}}(\mathrm{v})=\infty$

$\sum_{N-1} \operatorname{distG}(\mathrm{v})+\mathrm{TWG}(\mathrm{e}, \mathrm{v})<\operatorname{distG}(\mathrm{e}), \sum_{1}^{N-1} e$ update $\operatorname{distG}(\mathrm{e})$, where TWG is the $e=1, v=1$

Torrent Weight in the MWCBT framework. Therefore, this design can be considered as a greedy algorithm after computing $\mathrm{TW}_{\mathrm{G}}$. Hence, $\mathrm{TW}_{\mathrm{G}}$ uses local optimum to frame solution. As the algorithm proceeds, the minimum next vertex is explored in graph. This can be applied to a WSN field to find $\mathrm{TW}_{\mathrm{G}}$ where sensor nodes and links can be mapped.

\section{Experimental Setup}

In this section, the effectiveness of using MWCBT to eliminate congestion in WSNs is discussed. There are three performance metrics considered to predict the less congested path 
in a WSN namely packet drop rate, network throughput and average end-to-end delay. The energy utilization is another important parameter to be accounted as it indicates the total number of active nodes used in a data transmission and the residual energy of a node to perform data transmission. However, energy is not considered as a separate parameter for our analysis.

\subsection{Simulation environment}

In this segment, simulation is done using network simulator-2 version 2.35 . The network framework consists of 50 nodes deployed in $100 \mathrm{~m} * 100 \mathrm{~m}$ uniformly. The sensor nodes are supplied with omnidirectional antennas with a data buffer capacity of 60 packets. A common sharing of the wireless channel in a WSN is enabled by using the 802.11 protocol. The other required parameters are shown in the below Table 1.

Table 1. Simulation parameters

\begin{tabular}{llll}
\hline Parameters & Values & Parameters & Values \\
\hline Number of nodes & $50-100$ & Transmission range & $100 \mathrm{~m}$ \\
Idle power & $0.02 \mathrm{~W}$ & Transmission power & $2.5 \mathrm{~W}$ \\
Bandwidth & $850 \mathrm{Mb} / \mathrm{s}$ & Receiving power & $0.04 \mathrm{~W}$ \\
Data packet size & $512 \mathrm{bytes}$ & Number of sink(s) & 1 \\
Initial node energy & $3 \mathrm{~J}$ & Sleep power & $0.5 \mathrm{~mW}$ \\
Simulation time & $300 \mathrm{~s}$ & & \\
& & & \\
\hline
\end{tabular}

\subsection{Simulation outcomes}

The above MWCBT framework was compared with two important approaches namely M/Pareto [4][15] and Poisson[18] processes. The performance levels of M/Pareto and Poisson models are evaluated at various time intervals. The amount of packets sent by the source node to sink remains the same. There is a difference in the received packets by sink due to heavy arrival rate resulting in data congestion. The performance of MWCBT is evaluated at different time intervals during packet transmission. Figures 4 and 5 show MWCBT can handle data congestion at a better level by balancing energy level and minimizing packet loss rate. The proposed design draws more data packets from source node compared with M/Pareto and Poisson stochastic arrival processes. MWCBT receives more number of packets sent within the interval time of 300 s for 50 nodes used. Moreover, this model can extend receiving greater number of packets for the usage of 100 nodes within lesser interval time. 
In the proposed MWCBT framework, there is lower proportion of packet drop compared to other methods as seen in Table 2. The poisson queue modeling is prone to a maximum loss of more than 100 packets/s for a burst time of 90 ms. Energy consumption gradually increases from $0.25 \mathrm{~J} / \mathrm{s}$ to $0.46 \mathrm{~J} / \mathrm{s}$ for a burst time interval ranging from 5-30 s. The residual energy analysis of MWCBT with other prototypes with respect to delay and energy parameters is encapsulated in Table 3.

Table 2. Comparison of possible packet loss in MWCBT and other algorithms within burst time intervals.

\begin{tabular}{|r|r|r|r|r|}
\hline $\begin{array}{l}\text { Burst Time } \begin{array}{l}\text { B } \\
\text { (ms) }\end{array} \\
\text { r }\end{array}$ & Proposed MWCBT & Pareto Model [4] & $\begin{array}{l}\text { Pre-emptive } \\
\text { Multiple Queue } \\
{[20]}\end{array}$ & Poisson Model [12] \\
\hline 15 & 8 & 15 & 16 & 25 \\
\hline 30 & 17 & 21 & 28 & 37 \\
\hline 45 & 34 & 39 & 62 & 52 \\
\hline 60 & 32 & 46 & 85 & 75 \\
\hline 75 & 63 & 59 & 97 & 91 \\
\hline 90 & 70 & 88 & 105 \\
\hline
\end{tabular}

Table 3. Energy consumption in MWCBT algorithm within burst time intervals.

\begin{tabular}{|c|c|c|c|c|}
\hline \multirow{2}{*}{$\begin{array}{l}\text { Burst Time } \mathrm{B}_{\mathrm{t}} \\
\text { (s) }\end{array}$} & \multicolumn{2}{|c|}{ Time Utilization } & \multirow{2}{*}{$\begin{array}{l}\text { Delay Ds } \\
\text { (s) }\end{array}$} & \multirow{2}{*}{$\begin{array}{l}\text { Energy Es } \\
(\mathrm{J} / \mathrm{s})\end{array}$} \\
\hline & Initial RTT $\left(\mathrm{T}_{\mathrm{i}}\right)$ & $\begin{array}{l}\text { Service Time Delay } \\
\left(\mathrm{T}_{\mathrm{d}}\right)\end{array}$ & & \\
\hline 5 & 2 & 1 & 0.4 & 0.25 \\
\hline 10 & 4 & 3.5 & 0.47 & 0.29 \\
\hline 15 & 10 & 4.2 & 0.7 & 0.37 \\
\hline 20 & 14 & 7.8 & 0.64 & 0.32 \\
\hline 25 & 19 & 9.1 & 0.67 & 0.41 \\
\hline 30 & 25 & 12.5 & 0.66 & 0.46 \\
\hline
\end{tabular}

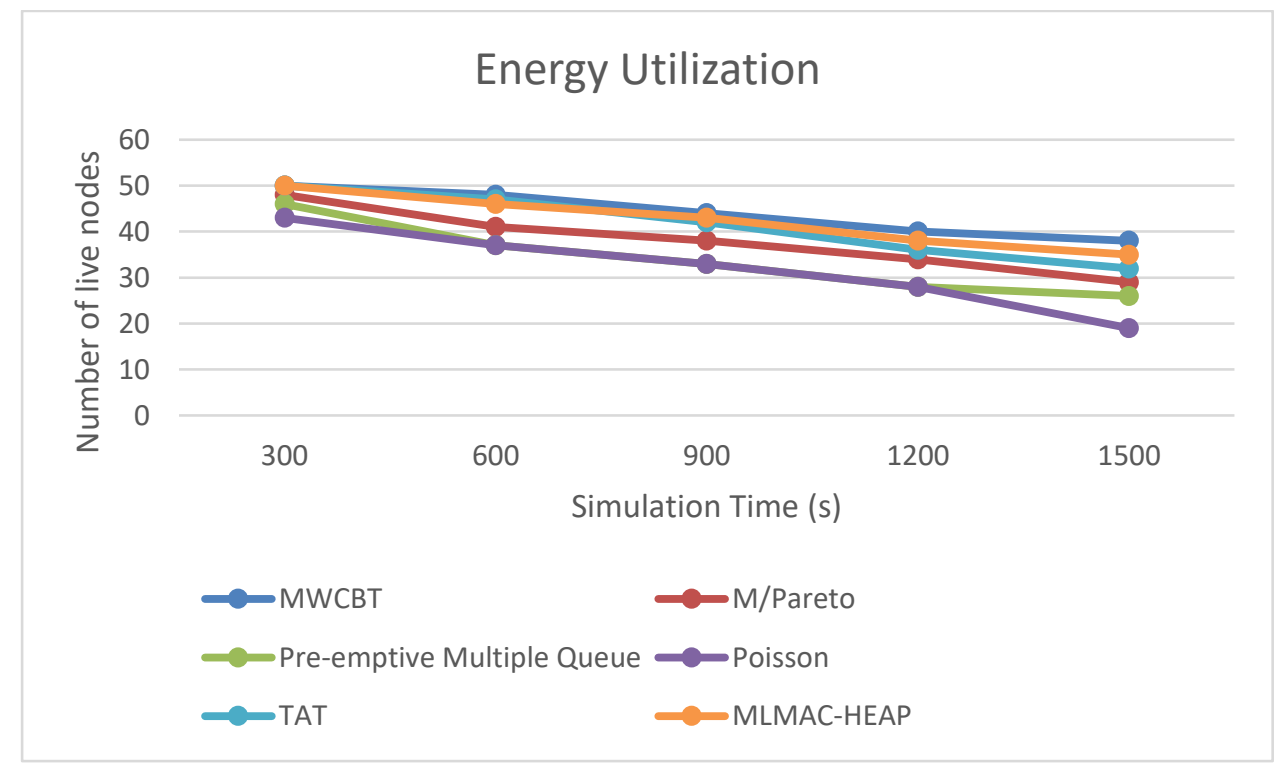


Fig.4. Residual energy in the sensor nodes

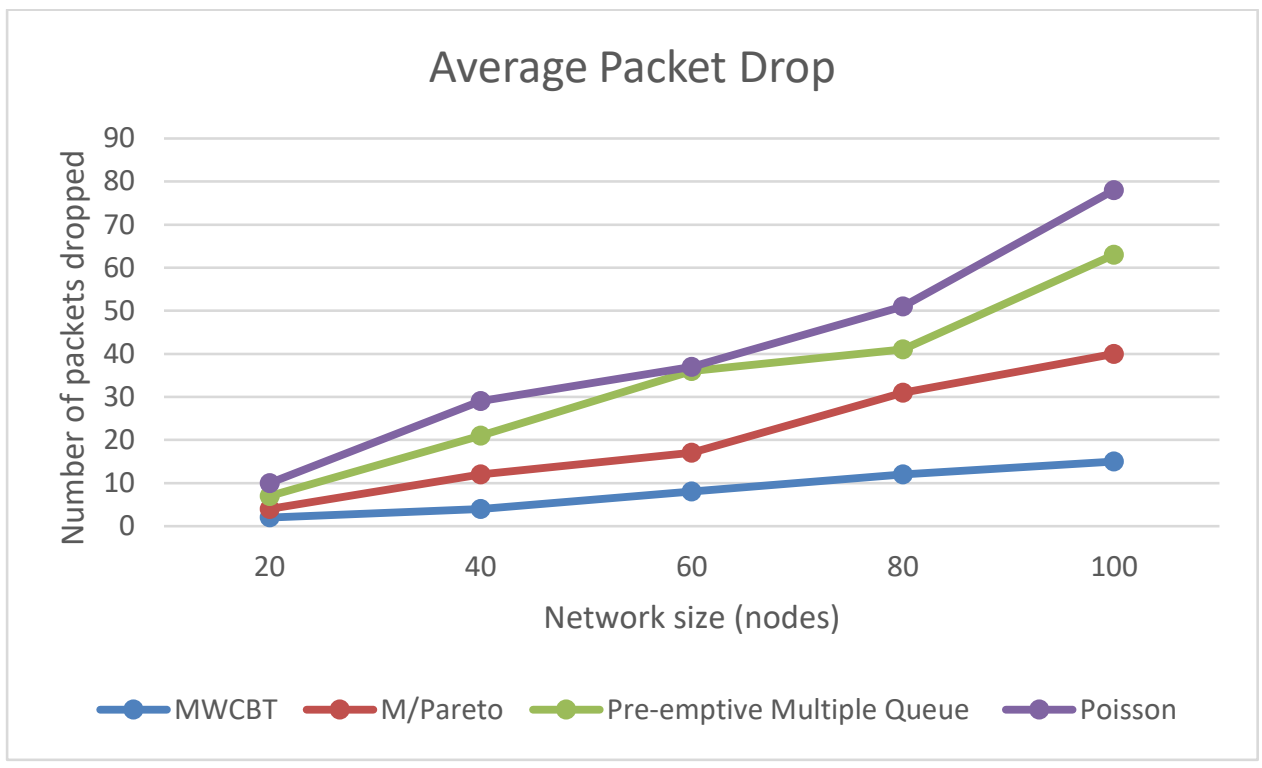

Fig.5. Overall packet loss due to heavy congestion causing network overhead

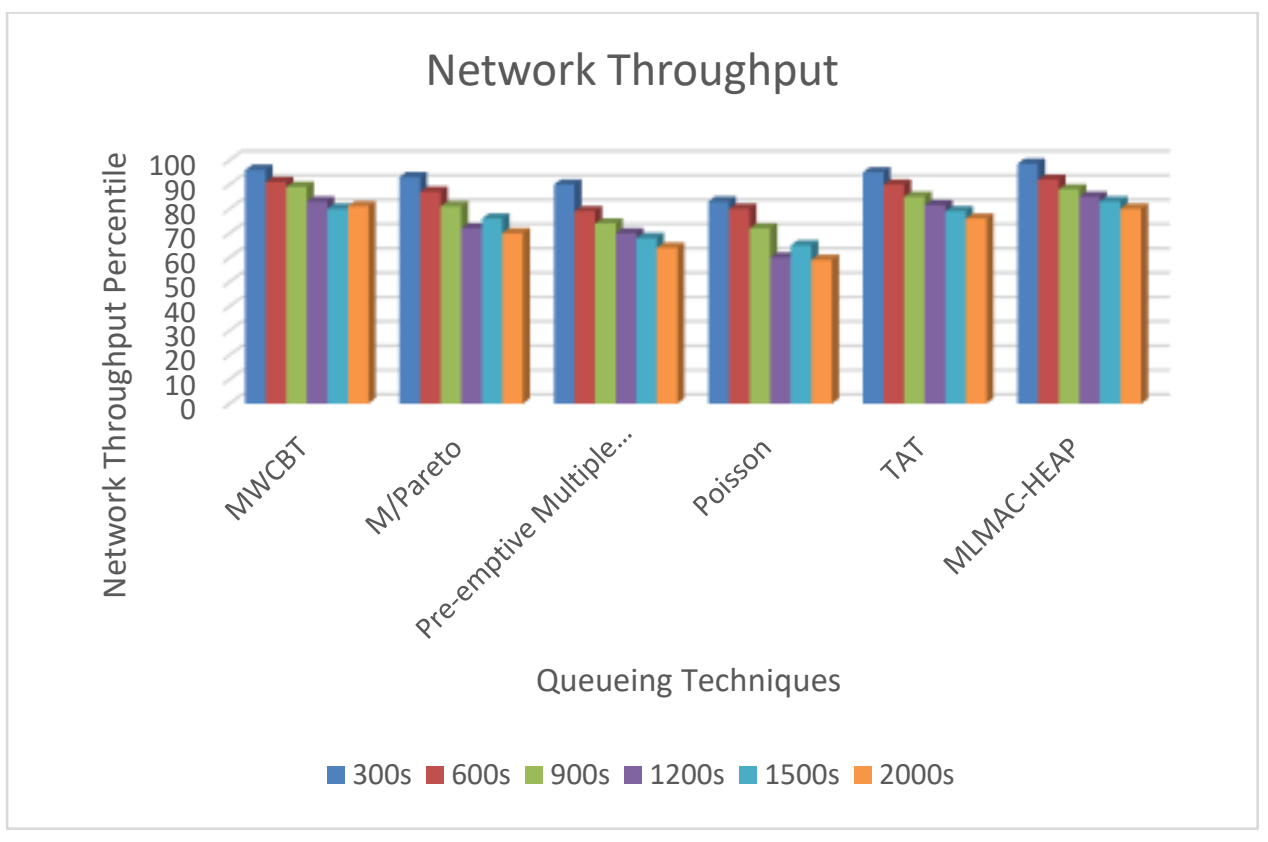

Fig.6. Overall network throughput

The results of overall energy usage, packet loss and network performance are shown using Figs. 4, 5 and 6. From [4], allowed congestion on boundary level with the period of congestion taking place in MWCBT system is shown in Table 4. If three factors taken into consideration such as mean, standard deviation and traffic rate constant, then the total congestion across channel for a time can be given using below tabular estimates. This table shows an allowed congestion constant that can be chosen for an incoming traffic rate. The congestion value $\mathrm{K}_{\mathrm{T}}$ is 
a constant that can be applied with mean and standard deviation to obtain channel capacity of a network link. A traffic distribution of the proposed algorithm with source arrival rate and packet utilization is listed in Table 5. There is a gradual increase in the energy and delay parameters as the packet arrival rate increases.

Table 4. Congestion rate in MWCBT algorithm within burst time intervals.

\begin{tabular}{|l|l|}
\hline Permissible congestion rate $\left(\mathrm{y}_{\mathrm{C}}\right)$ & Time congestion constant $\left(\mathrm{K}_{\mathrm{T}}\right)$ \\
\hline 0.2 & 2.56 \\
\hline 0.021 & 3.21 \\
\hline 0.003 & 4.08 \\
\hline $10-5$ & 4.83 \\
\hline $10^{-6}$ & 5.07 \\
\hline $10^{-7}$ & 5.64 \\
\hline
\end{tabular}

Table. 5. Comparison of Traffic Distribution between MWCBT algorithm and other algorithms

\begin{tabular}{|c|c|c|c|c|c|c|c|c|c|c|}
\hline \multirow{2}{*}{$\begin{array}{l}\text { Process } \\
\text { Arrival } \\
\text { Rate } \\
(\mathrm{Mb} / \mathrm{s})\end{array}$} & \multirow[b]{2}{*}{$\begin{array}{l}\text { Traffic } \\
\text { Intensi } \\
\text { ty }\end{array}$} & \multicolumn{4}{|c|}{ Less traffic scenario } & \multicolumn{5}{|c|}{ Excess traffic scenario (Congestion state) } \\
\hline & & $\begin{array}{l}\text { Packet } \\
\text { utilizati } \\
\text { on } \\
\text { probabil } \\
\text { ity (pps) }\end{array}$ & $\begin{array}{l}\text { No. of } \\
\text { packets } \\
\text { transferre } \\
\text { d }\end{array}$ & $\begin{array}{l}\text { Residu } \\
\text { al } \\
\text { Energy } \\
(\mathrm{J} / \mathrm{s})\end{array}$ & $\begin{array}{l}\begin{array}{l}\text { Delay } \\
\text { (ms) }\end{array}\end{array}$ & $\begin{array}{l}\text { Traffic } \\
\text { Intensit } \\
\mathrm{y}\end{array}$ & $\begin{array}{l}\text { Packet } \\
\text { utilizatio } \\
\text { n } \\
\text { probabili } \\
\text { ty (pps) }\end{array}$ & $\begin{array}{l}\text { No. of } \\
\text { packe } \\
\text { ts } \\
\text { transf } \\
\text { erred }\end{array}$ & $\begin{array}{l}\text { Residual } \\
\text { Energy } \\
(\mathrm{J} / \mathrm{s})\end{array}$ & $\begin{array}{l}\text { Delay } \\
(\mathrm{ms})\end{array}$ \\
\hline 125 & 0.14 & 0.37 & 14000 & 0.13 & 0.5 & 1.9 & 0.005 & 10500 & 0.16 & 5.1 \\
\hline 250 & 0.29 & 0.75 & 28495 & 0.18 & 1.2 & 2.4 & 0.007 & 8400 & 0.28 & 11.58 \\
\hline 375 & 0.44 & 1.12 & 35700 & 0.24 & 2.5 & 2.86 & 0.008 & 14650 & 0.45 & 16 \\
\hline 500 & 0.58 & 1.5 & 49200 & 0.3 & 3.1 & 4.3 & 0.012 & 12800 & 0.7 & 23.7 \\
\hline 625 & 0.73 & 1.87 & 60500 & 0.38 & 4 & 7.75 & 0.023 & 17000 & 1.02 & 30.5 \\
\hline 750 & 0.88 & 2.25 & 72000 & 0.41 & 6.5 & 10.2 & 0.03 & 25400 & 1.35 & 45 \\
\hline
\end{tabular}




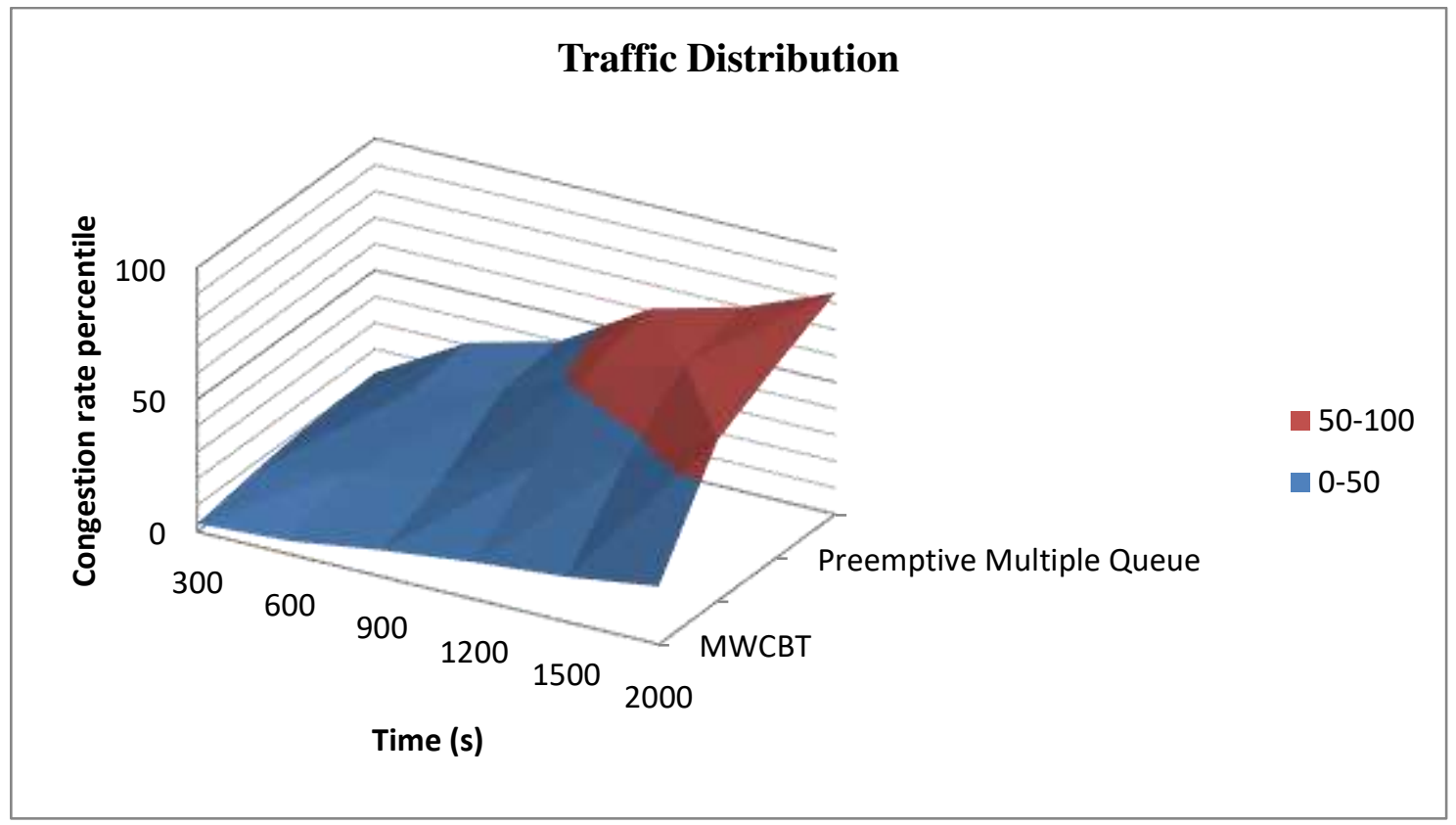

Fig. 7. Comparison of congestion distribution at different time intervals for other stochastic processes with the proposed MWCBT design.

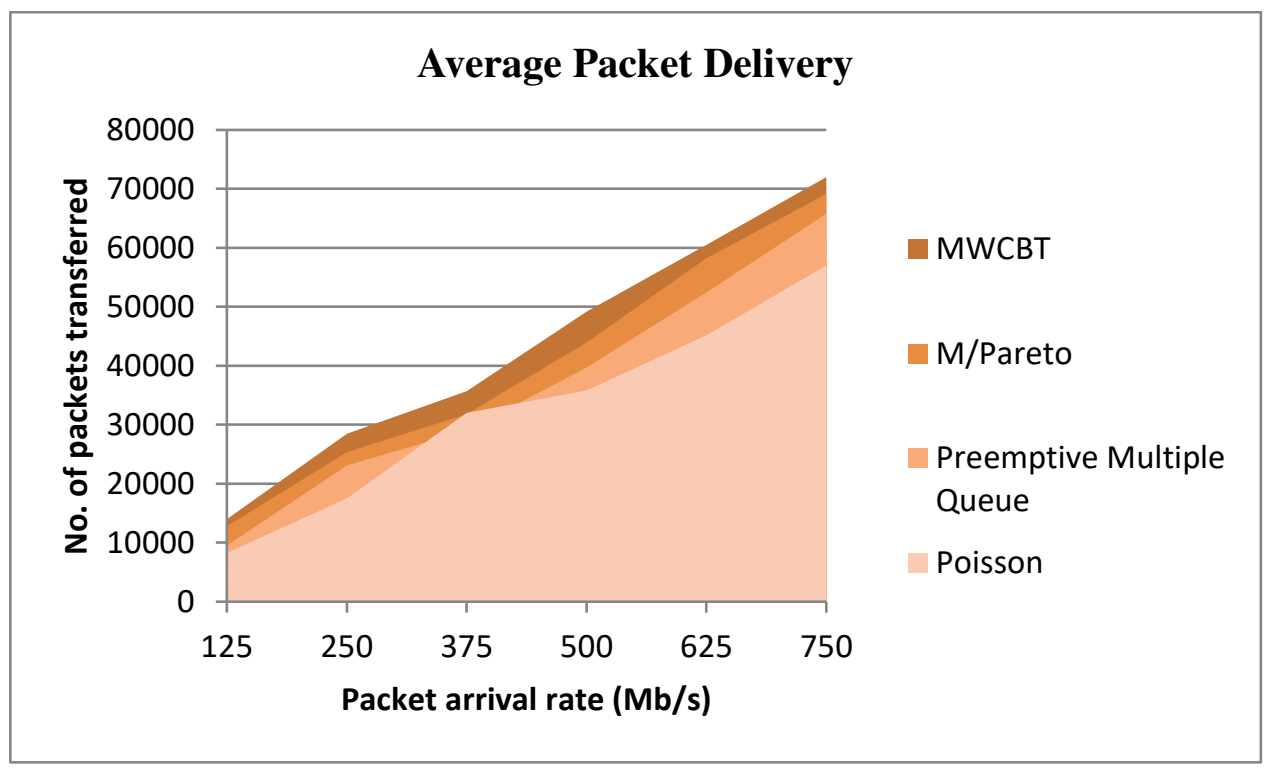

Fig.8. Overall proportion of successfully received data packets received in sink when maximum source data rate is 140 .

Fig. 7 illustrates the average congestion percentage for the queuing modeled techniques for an interval ranging from $300 \mathrm{~s}$ to $2000 \mathrm{~s}$. The maximum packet transfer in different stochastic models is indicated in Fig. 8. At the end of an arrival rate $750 \mathrm{Mb} / \mathrm{s}, \mathrm{MWCBT}$ accomplishes a packet transfer of 72000 packets, followed by Pareto, Preemptive Multiple Queue and Poisson 
with 69150,65774 and 57025 packets. This shows that there is a considerable increased packet transport within buffer queue in MWCBT algorithm.

Table 6. Mean queue length between MWCBT and other algorithms

\begin{tabular}{|l|l|l|l|l|}
\hline \multirow{2}{*}{$\begin{array}{l}\text { No. of CBR } \\
\text { connections }\end{array}$} & \multicolumn{4}{|c|}{ Average Queue Length } \\
\cline { 2 - 5 } & Proposed MWCBT & Pareto Model [4] & $\begin{array}{l}\text { Pre-emptive } \\
\text { Multiple Queue } \\
{[20]}\end{array}$ & Poisson Model [12] \\
\hline 5 & 10.751 & 12.950 & 13.508 & 17.835 \\
\hline 10 & 10.264 & 12.310 & 13.200 & 17.502 \\
\hline 20 & 9.570 & 11.860 & 12.790 & 16.150 \\
\hline 30 & 8.735 & 11.130 & 12.220 & 15.466 \\
\hline 40 & 8.226 & 10.557 & 11.441 & 14.670 \\
\hline 50 & 6.199 & 9.632 & 10.425 & 13.564 \\
\hline
\end{tabular}

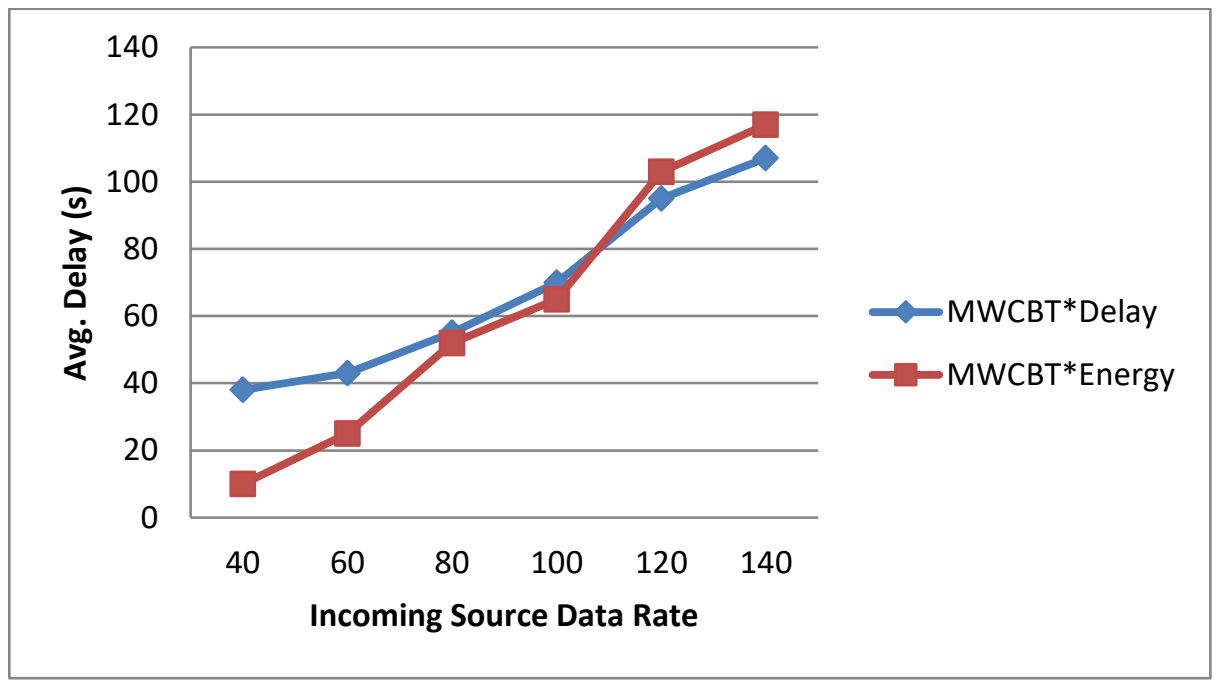

Fig.9. Average end-to-end Time Delay and Energy usage

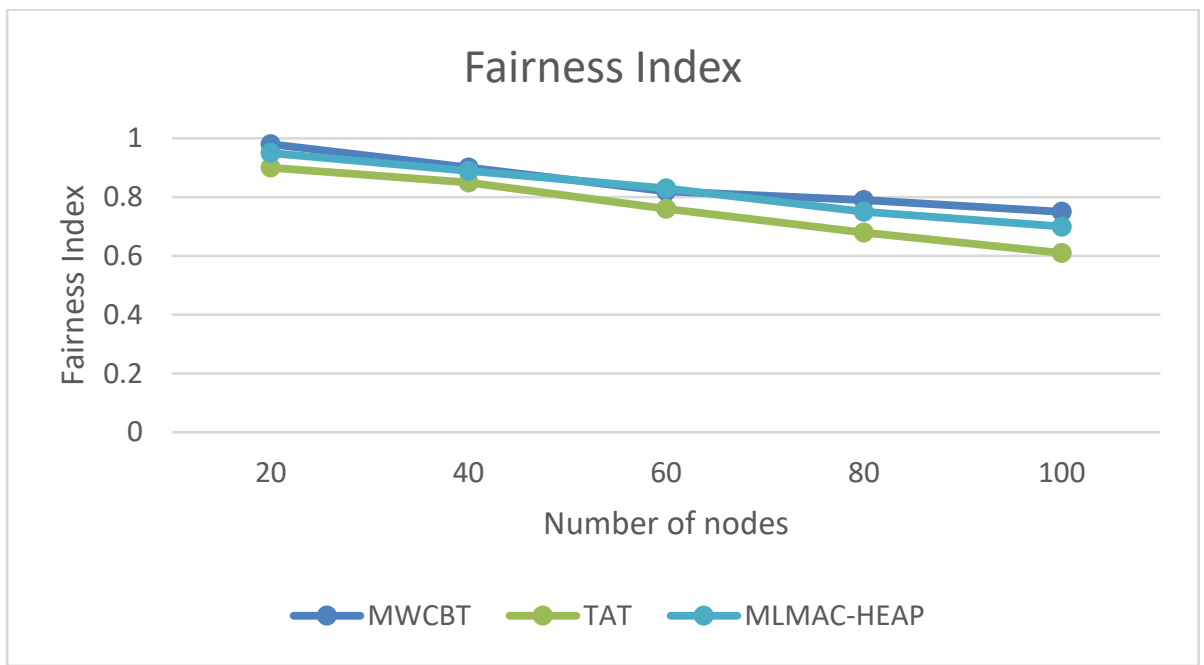

Fig.10. Fairness Index for data delivery in different techniques 


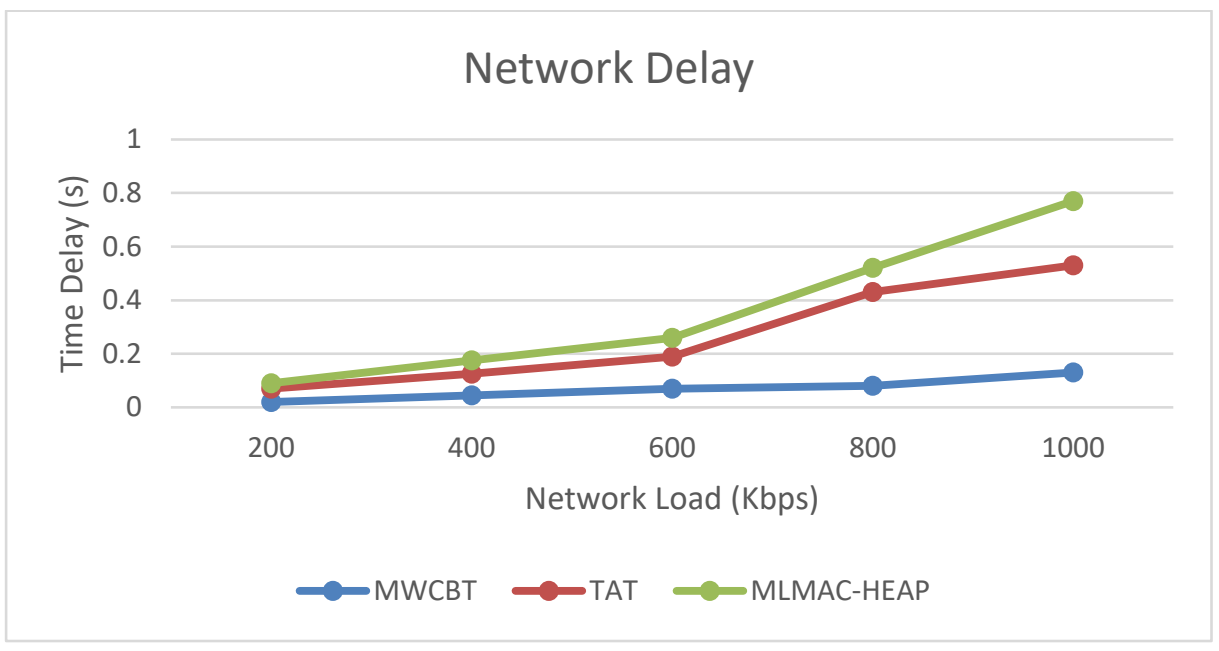

\section{Fig.11. Comparison of network delay in various methodologies}

Figures 10 and 11 denote the fairness and network delay occurring in various queueing techniques. Table 6 gives a description of the average queue length between MWCBT and other system models. From Figure 5, it can be seen that MWCBT reduces packet drop proportion upto $28 \%$ and $40 \%$ when compared with M/Pareto and Poisson. This projects that the proposed framework is effective to improve the overall network performance. To check congestion, MWCBT starts executing the computation of torrent weight and choosing the accurate corrected path to sink from initial stage of packet transfer. This lessens the likelihood of packet loss in MWCBT. The proposed design is found to lower the average end-to-end latency to improve the network performance. Figure 6 demonstrates that the highest throughput of MWCBT is $98 \%$, whereas M/Pareto shows $94 \%$ and Poisson exhibits $86 \%$ for a network size of 10 nodes. This MWCBT approach increases the network throughput by 7-20\% compared with M/Pareto and Poisson as shown in fig. 4. Figures 7 and 8 illustrate the percentage of congestion between nodes and total number of successfully received packets in the sink node. Figure 9 visualizes the time delay involved to transmit packets using MWCBT from source to sink nodes.

In order to find out the capability of MWCBT in handling energy consumption, the average residual energy is estimated for each live node in the sensor network after a cycle of 300s with 50 nodes. The residual energy comparison for different models is shown in Figure 4. The sink is located at $(95,0)$. The activity of power leakage can proceed to continue throughout the sensor network span from less to extreme cases. This depicts that the proposed framework lowers the energy consumed by the entire range of nodes in the sensor network. 


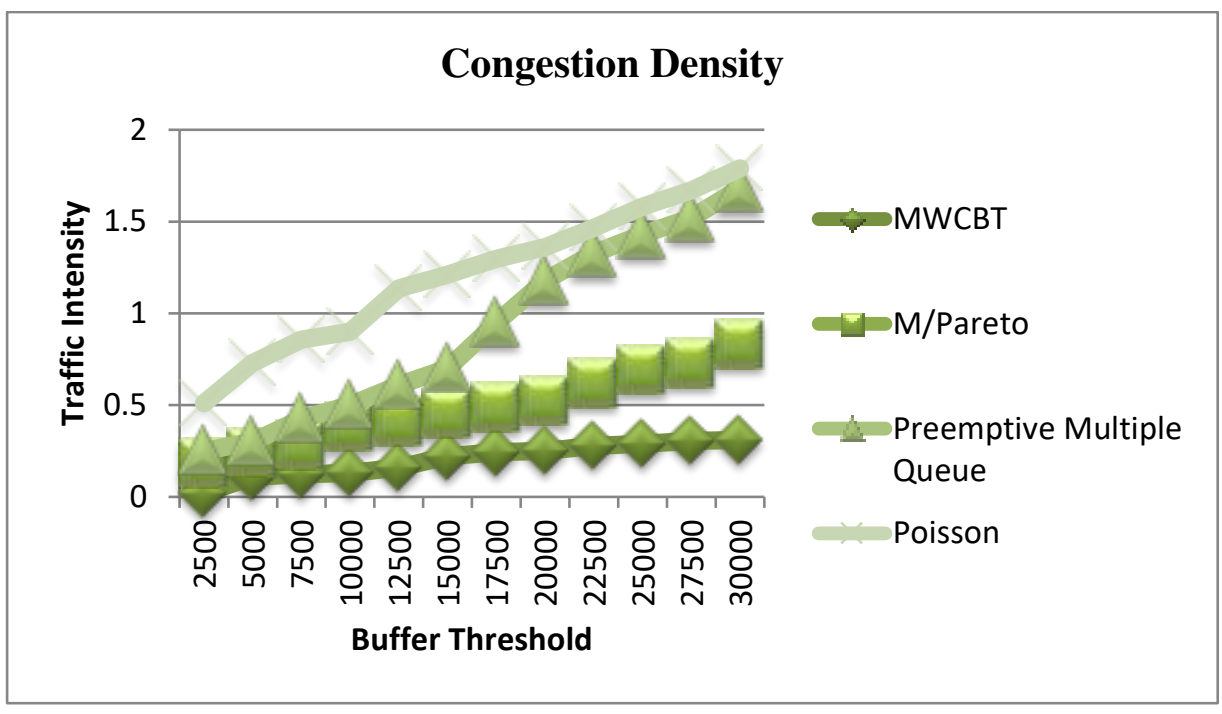

Fig. 12. Congestion Density between MWCBT algorithm and other algorithms

Figure 12 illustrates the rate of congestion for an input buffer of acceptable packet flow. For a buffer limit of 2500 pps, it can be observed that MWCBT achieves least congestion value of 0.1 and for $30000 \mathrm{pps}$, the algorithm achieves a lower value of 0.31 . When compared with the other algorithms, MWCBT involves less overhead in terms of packet congestion. The poisson method of queuing suffers a greater packet collision of 1.7 and higher for an input buffer flow of 30000 pps.

In the proposed MWCBT mechanism, there is a considerable increase in delay range of $0.03 \mathrm{~s}$ at the end of every round for 200s simulation. Hence, there is an increase of $8 \%$ delay during data transmission from nodes when compared to the other existing techniques. The net control overhead occurring in a node is given as the ratio between control information transmitted by the node and actual data received in each node. An increase of $12 \%$ control overhead is faced by MWCBT in comparison to other existing techniques after a simulation of 350 rounds. As the scalability of nodes increase, there is an increase of control overhead in overall network.

The proposed MWCBT takes a computational complexity of $\mathrm{O}(\mathrm{n})$ for incoming packets. For $\mathrm{n}$ nodes in network, each node estimates the next better hop based on calculated torrent weight. Each node is aware of torrent weight in neighbor nodes, hence the weight of the independent nodes in entire network nodes need to be computed. There are n-1 times of node visits occurring to predict optimal path of least weighted nodes in network. Thus, the total time complexity for $\mathrm{n}$ nodes is $\mathrm{O}(\mathrm{n})$. The time complexity of Poisson queueing and Pareto queueing processes is given as $\mathrm{O}(\log \mathrm{n})$. The arrival in node buffer queue is divided into two subgroups such as 
minimum and maximum threshold limits. The node buffer is checked for $n-p$ and $n-q$ ends to find whether packet load falls below or above threshold ranges.

\section{Conclusions}

This paper has depicted the use of M/Pareto process in estimating the maximum number of data packets incoming to a sensor node. The proposed MWCBT method uses M/Pareto stochastic process to accurately predict the network traffic stream. The network measures such as energy and time delay that affect the packet buffer on a node were also considered for the prediction of torrent weight. From the above results, this MWCBT approach is found to perform accurately and yield a better possible solution to congestion in WSNs. The proposed approach has a maximum delay of 0.13 at an applied network load of $1000 \mathrm{Kbps}$. For a simulation time of 2000s, the MWCBT traffic model achieves a maximum throughput of 81 percentile that is greater than the other existing mechanisms. Moreover, there are only 15 packets dropped on addition of 100 nodes to network.

In the future research, the MWCBT framework can be extended with other stochastic process distributions such as Random walks, Markov processes and Gaussian processes. Several other network parameters can be tuned to achieve maximum network model efficiency. Functioning of the proposed mechanism on integration with resource control algorithms and their performance results need to be evaluated. These distributions can be compared with the proposed congestion mitigation modeling with various mean packet arrival rates. An outlined study of efficiently testing the above listed stochastic processes to countercheck congestion in extreme torrent cases need to be resolved. Therefore, it is necessary to give a well-formed traffic model operating during excess traffic conditions with minimal packet loss and has a good ability to function in case of parallel and distributed network supported environments.

\footnotetext{
*There are no funding resources supporting this research work.

* Data sharing is not applicable to this article as no datasets were generated or analyzed during the current study.
}

* On behalf of all authors, the corresponding author states that there is no conflict of interest. 


\section{$10 \quad$ References}

1. O. Chughtai, N. Badruddin, A. Awang, M. Rehan, "Congestion-aware and traffic load balancing scheme for routing in WSNs", Telecommunication Systems, Vol. 63, Iss. 4, pp 481-504, 2016.

2. C. Sergiou, P. Antoniou,V. Vassiliou, "A Comprehensive Survey of Congestion Control Protocols in Wireless Sensor Networks", IEEE Communication Surveys and Tutorials, Vol. 16, No.4, Fourth Quarter, pp. 1839-1859, 2014.

3. K. Murugan, S. Shanmugavel, "Traffic-Dependent and Energy-Based Time Delay Routing Algorithms for Improving Energy Efficiency in Mobile Ad Hoc Networks", EURASIP Journal on Wireless Communications and Networking, Vol. 5, pp. 625-634, 2005.

4. T. D. Neame, M. Zukerman, R. G. Addie, "Application of the M/Pareto Process to Modeling Broadband Traffic Streams", IEEE Proceedings of ICON 99', pp. 53-58, 28 September-1 October, Brisbane, Queensland, Australia,1999.

5. M. Zukerman, T. D. Neame, G. Addie, "Internet Traffic Modeling and Future Technology Implications", IEEE INFOCOM, Twenty-Second Annual Joint Conference of the IEEE Computer and Communications, 2003.

6. M. Hosamo, "Source Traffic Modeling Using Pareto Traffic Generator", Journal of Computer Networks, Vol. 4, No. 1, pp. 11-19, 2017.

7. R. G. Addie, M. Zukerman, T. D. Neame, "Broadband Traffic Modeling: Simple Solutions to Hard Problems", IEEE Communications Magazine, pp. 88-95, 1998.

8. S. Tanga, L. Tan, "Single-wavelength optical buffers with general burst size distribution: Blocking probability and mean delay", Optical Switching and Networking, Vol. 27, pp. 16, 2018.

9. O. Markelov, V. N. Duc, M. Bogachev, "Statistical modeling of the Internet traffic dynamics: To which extent do we need long-term correlations?", Physica A, Vol. 485, pp. 48-60, 2017.

10. B. A. Peters, J. S. Smith, D. J. Medeiros, and M. W. Rohrer, "Using quantile estimates in simulating internet queues with Pareto service times", Proceedings of the Winter Simulation Conference, pp. 477-485, 2002.

11. N. G. Duffield, W. Whitt, "Network Design and Control Using ON/OFF and Multilevel Source Traffic Models with Heavy-Tailed Distributions", Self-Similar Network Traffic and Performance Evaluation, Copyright 2000 John Wiley \& Sons, Inc 
12. S. Feng, "The Poisson-Dirichlet Distribution and Related Topics: Models and Asymptotic Behaviors, Springer-Verlag Berlin Heidelberg, 2010.

13. Floyd, V. Paxon, "Wide-Area Traffic: The failure of Poisson Modeling", Proc. ACM Sigcomm, London, UK, pp. 257-268, 1994.

14. S. Foss, D. Korshunov, S. Zachary, An Introduction to Heavy-Tailed and Subexponential Distributions [2 ed.], Springer-Verlag New York, 2013.

15. B. C. Arnold, Pareto Distributions Second Edition, Taylor and Francis, 2015.

16. A. Feldmann, A. C. Gilbert, W. Willinger, T. G. Kurtz,"The changing nature of network traffic: Scaling phenomena", ACM SIGCOMM Computer Communication Review, pp. 529, Vol. 28, Issue 2, 1998.

17. K. Briggs, C. Beck, "Modelling train delays with q-exponential functions", Physica A, pp. 498-504, Vol. 378, Issue 2, 2007.

18. M. Zukerman, "Introduction to queueing theory and stochastic teletraffic models", 2013. ArXiv preprint arXiv:1307.2968.

19. J. Huang, D. Du, Q. Duan, Y. Zhang, Y. Zhao, H. Luo, Z. Mai, Q. Liu,"Modeling and Analysis on Congestion Control for Data Transmission in Sensor Clouds", International Journal of Distributed Sensor Networks, Hindawi Publishing Corporation, pp.1-9, 2014.

20. G. P. Sunitha, S. M. Dilip Kumar, B. P. Vijay Kumar,"A Pre-emptive Multiple Queue based Congestion Control for Different Traffic Classes in WSN", Proceedings of International Conference on Circuits, Communication, Control and Computing, pp. 212218, 2014.

21. I.F. Akyildiz, W. Su, Y. Sankarasubramaniam, E. Cayirci,"Wireless sensor networks: a survey", Elsevier, Journal on Computer Networks, Vol. 38, pp. 393-422, 2002.

22. P. Antoniou, A. Pitsillides, T. Blackwell, A. Engelbrecht, L. Michael,’Congestion control in wireless sensor networks based on bird flocking behavior", Elsevier, Journal on Computer Networks, Vol. 57, pp. 1167-1191, 2013.

23. S. Brahma, M. Chatterjee, K. Kwiat, P. K. Varshney,"Traffic management in wireless sensor networks: Decoupling congestion control and fairness", Elsevier, Journal on Computer Communications, Vol. 35, pp. 670-681, 2012.

24. C. Sergiou, V. Vassiliou, A. Paphitis,"Congestion control in Wireless Sensor Networks through dynamic alternative path selection", Elsevier, Journal on Computer Networks, Vol. 75, pp. 226-238, 2014. 
25. S. Lall, A. S. Alfa, B. T. Maharaj, "The Role of Queueing Theory in the Design and Analysis of Wireless Sensor Networks: An Insight", IEEE 14th International Conference on Industrial Informatics (INDIN), pp. 1191-1194, 2016.

26. J. Jin, M. Palaniswami, B. Krishnamachari,"Rate control for heterogeneous wireless sensor networks: Characterization, algorithms and performance", Elsevier, Journal on Computer Networks, Vol. 56, pp. 3783-3794, 2012.

27. T. Dong, W. Hu, X. Liao,"Dynamics of the congestion control model in underwater wireless sensor networks with time delay", Elsevier, Journal on Chaos, Solitons and Fractals, Vol. 92, pp. 130-136, 2016.

28. E. Stai, S. Papavassiliou,"User Optimal Throughput-Delay Trade-off in Multihop Networks Under NUM Framework", IEEE Communications Letters, Vol. 18, No. 11, pp. 1999-2002, 2014.

29. A. F. Agamy, A. M. Mohamed, "Performance Modeling of WSN with Bursty Delivery Mode", Cornell University, arXiv:1702.03907 [cs.NI], pp.1-12, 2017.

30. P. Wang, I. F. Akyildiz, "Spatial Correlation and Mobility-Aware Traffic Modeling for Wireless Sensor Networks", IEEE/ACM Transactions on Networking, Vol. 19, Iss. 6, pp. 1860 - 1873, 2011.

31. F. Kauer, V. Turau, "An analytical model for wireless mesh networks with collision-free TDMA and finite queues", EURASIP Journal on Wireless Communications and Networking, Vol. 149, pp. 1-17, 2018.

32. A. A. Halafi, A. Alghadhban, B. Shihada, "Queuing Delay Model for Video Transmission Over Multi-Channel Underwater Wireless Optical Networks", IEEE Access, Vol. 7, pp. 10515 - 10522, 2019.

33. S. A. Shah, B..Nazir, I. A. Khan, "Congestion Control algorithms in wireless sensor networks: Trends and opportunities”, Elsevier, J. of King Saud University - Computer and Information Sciences, pp. 1319-1578, 2016.

34. H. Tall, G. Chalhoub, N. Hakem, M. Misson, "Load balancing routing with queue overflow prediction for WSNs", Wireless Networks, Vol. 25, Iss. 1, pp. 229-239, 2019.

35. D. P. Kumar, T. Amgoth, C. S. R. Annavarapu, "Machine learning algorithms for wireless sensor networks: A survey", Information Fusion, Vol. 49, pp. 1-25, 2019.

36. P. K. Donta, T. Amgoth and C. S.R. Annavarapu, "Congestion-aware Data Acquisition with Q-learning for Wireless Sensor Networks", IEEE International IOT, Electronics and Mechatronics Conference (IEMTRONICS), 9-12 Sept. 2020. 
37. A. Kochhar, P. Kaur, P. Singh, B. S. Sohi, "MLMAC-HEAP: A Multi-Layer MAC Protocol for Wireless Sensor Networks Powered by Ambient Energy Harvesting", Wireless Personal Communications, vol. 110, pp. 893-911, 2020.

38. A. Kak, A. Kureev, E. Khorov, I. F. Akyildiz, "Radio access network design with softwaredefined mobility management", Wireless Networks, Vol. 26, pp.3349-3362, 2020.

39. B. Khandish, J. Suk, "A Network Traffic Aware Tuning Algorithm in Wireless Sensor Networks", Wireless Personal Communications, 118, 431-445, 2021.

40. X. Li, F. Ren,B. Yang, "Modeling and Analyzing the Performance of High-Speed Packet I/O", Tsinghua Science and Technology, Vol. 26 Issue. 4, 426 - 439.

41. D. K. Sah, K. Cengiz, P. K. Donta, V.N.Inukollu, T. Amgoth, "EDGF: Empirical dataset generation framework for wireless sensor networks", Computer Communications, Vol. 180, pp. 48-56, 2021. 